\title{
Composición de semillas de girasol cultivadas en Argentina
}

\author{
Por E. Santalla, I. Riccobene y S. Nolasco \\ Facultad de Ingeniería de la Universidad Nacional del Centro de la Provincia de Buenos Aires. \\ Avda. A. del Valle 5737, 7400-Olavarría, Buenos Aires (Argentina).
}

\section{RESUMEN}

Composición de semillas de girasol cultivadas en Argentina.

Se evaluaron contenidos de aceite de los constituyentes de la semilla, estructura física, calidad del aceite de semilla y contenido de ceras.

El conocimiento del comportamiento de estos caracteres, las relaciones entre ellos y su interacción con el medio agroclimático, facilita la introducción de este cultivo en el mercado alimenticio y/o industrial optimizando su rendimiento y aprovechamiento.

Se presentan los valores de las correlaciones obtenidas entre los caracteres analizados.

Se plantean hipótesis de causalidad sobre las nuevas tendencias de comportamiento de este cultivo.

Se destaca la influencia del contenido de cáscara tanto sobre el rendimiento del contenido de aceite como sobre la cantidad de ceras de la semilla, de las cuales se considera principal portadora.

PALABRAS-CLAVE: Aceite (calidad) - Argentina - Cera -Girasol (semilla)

\section{SUMMARY}

Compositional data on sunflower seed grown in Argentine.

Constituents seed oil content, physic structure, oil seed quality and wax content are evaluated.

The relations of these characters and its agroclimatic interactions facilitate the introduction of this cultivar into food and/or industrial market improving its utility and profit.

Correlations coefficients on the analyzed characters are presented.

Causality hypothesis on the newer tendency behaviour of this cultivar are planed.

The hull content influence on oil content and like principal wax container is detached.

KEY-WORDS: Argentine - Oil (quality) - Sunflower (seed) - Wax.

\section{INTRODUCCION}

El girasol, una de las principales oleaginosas que se cultiva en Argentina, tiene como mercado más importante la producción de aceites y derivados mientras que en menor proporción se utiliza la semilla sin procesar para la alimentación humana y animal.

La mejora genética llevada a cabo en los últimos años, ha logrado híbridos con mayores contenidos de aceite en la semilla pero paralelamente ha surgido el problema de una disminución del índice de extracción.

El incremento de la participación de la industria aceitera en el mercado exportador, obliga a mejorar la calidad de los aceites producidos y a explotar más intensamente este cultivo desde el punto de vista nutricional, lo que lleva a considerar la posiblidad de alcanzar un mayor conocimiento sobre la influencia que factores climatológicos, como la temperatura, ejercen sobre la calidad del aceite de este cultivo, específicamente sobre algunos ácidos grasos.

La utilización del girasol en función de su futura ubicación en el mercado, induce a conocer sus caracteres más importantes, lo que implica determinar cómo se relacionan entre sí e interaccionan con el medio agroclimático.

A tal fin, se calcularon los coeficientes de correlación entre: rendimiento (contenido de aceite por semilla), contenidos de cáscara y de pipa, contenidos de aceite de cáscara y de pipa, calidad del aceite de la semilla y contenido de ceras.

\section{PARTE EXPERIMENTAL}

Se trabajó con los siguientes híbridos de girasol, correspondientes a las cosechas 1984/85 y 1989/90: Súper (S)400 , S-401, S-405, S-406, S-407, S-430, S-530, P-80, G90, Contiflor (CF), CF-3, 891 y Sungro (S)-380.

Las localidades argentinas en las cuales se cosecharon los cultivos estudiados fueron: Avia Terai (I), Reconquista (II), Río IV (III), Patricios (IV), Pigüe (V) y Necochea ( VI) .

Se separó en forma manual cáscara y pipa determinando gravimétricamente los porcentajes de las mismas. Se extrajeron con hexano (Soxhlet, $24 \mathrm{hs}$.) los aceites de semilla, de cáscara y de pipa y se evaluó la calidad del aceite total a través del análisis de su composición acídica (CGL, columna de acero inoxidable con relleno Chromosob W-AW (60-80\%) conteniendo $15 \%$ de adipato de etilenglicol polímero).

La cantidad de ceras se determinó según la técnica de lavado con hexano (1).

Para todos los análisis efectuados se tomó la misma base de cálculo: el peso de semilla seca. 


\section{RESULTADOS}

A continuación se presentan las correlaciones obtenidas entre las variables estudiadas. Los dos valores de "r" representan lo obtenido para las cosechas estudiadas; en adelante "Cos I": Cosecha 1984/85, y "Cos II": Cosecha 1989/90.

En el Gráfico № 1 se observa el comportamiento de los contenidos de cáscara y de pipa respecto de la cantidad de aceite de semilla.

Se observa que los híbridos de menor cantidad de cáscara contienen más aceite en la semilla $(r=-0.70$ y $r=-0.72)$, tendencia coherente con los valores de correlación positivos obtenidos para los caracteres aceite en pipa-cantidad de pipa ( $r=+0.72$ y $r=+0.62$ ), aceite en pipa-aceite total $(r=+0.70$ y $r=+0.72)$ y cantidad pipa-contenido de aceite total $(r=+0.68$ y $r=+0.63)$.

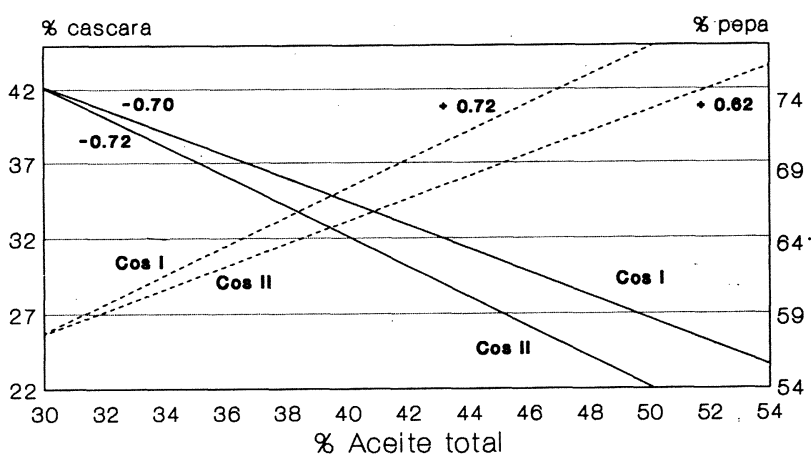

Híbridos: S-530, CF, CF3 (32,1\%-43,4\%)

S-401/405/406/430/407,G-90 (40,3\%-47,6\%)

S-400, P-80, S-380, 891 (48,4\%-53,5\%)

Gráfico № 1

Contenidos de cáscara y pipa en función del aceite total.

Tabla 1

Datos de composición acídica.

(Esteres metilicos \% ésteres metílicos totales).

\begin{tabular}{|c|c|c|c|c|c|c|}
\hline \multicolumn{7}{|c|}{ COSECHA '84/85 } \\
\hline Localidad & Híbrido & $16: 0$ & $18: 0$ & $18: 1$ & 18:2 & $18: 1 / 18: 2$ \\
\hline \multirow[t]{4}{*}{ RECONQUISTA } & S-380 & 7.8 & 2.4 & 33.3 & 56.5 & 0.59 \\
\hline & 891 & 8.1 & 1.6 & 32.0 & 58.3 & 0.55 \\
\hline & S-401 & .7 .4 & 1.3 & 35.9 & 55.5 & 0.65 \\
\hline & CF & 7.5 & 1.6 & 40.4 & 50.5 & 0.80 \\
\hline \multirow[t]{4}{*}{ PATRICIOS } & S-380 & 7.2 & 2.4 & 24.6 & 65.8 & 0.37 \\
\hline & 891 & 7.1 & 2.5 & 27.0 & 63.3 & 0.43 \\
\hline & S-401 & 6.5 & 2.1 & 25.7 & 65.7 & 0.39 \\
\hline & CF & 6.8 & 1.3 & 26.5 & 65.4 & 0.41 \\
\hline \multirow[t]{4}{*}{ NECOCHEA } & S-380 & 7.6 & 1.5 . & 20.8 & 70.1 & 0.30 \\
\hline & 891 & 8.3 & 1.5 & 20.4 & 69.8 & 0.29 \\
\hline & S-401 & 7.8 & 1.8 & 19.4 & 72.1 & 0.27 \\
\hline & CF & 6.5 & 2.0 & 18.5 & 73.0 & 0.25 \\
\hline \multicolumn{7}{|c|}{ COSECHA ' $89 / 90$} \\
\hline Localidad & Híbrido & $16: 0$ & $18: 0$ & $18: 1$ & $18: 2$ & $18: 1 / 18: 2$ \\
\hline \multirow[t]{4}{*}{ AVIA TERAI } & S-407 & 7.3 & 1.8 & 35.7 & 55.2 & 0.65 \\
\hline & $S-430$ & 6.6 & 1.5 & 35.2 & 56.7 & 0.62 \\
\hline & S-530 & 7.4 & 1.6 & 41.3 & 49.7 & 0.83 \\
\hline & CF3 & 6.8 & 1.8 & 36.2 & 55.2 & 0.66 \\
\hline \multirow[t]{4}{*}{ RECONQUISTA } & S-407 & 7.8 & 1.1 & 35.7 & 55.3 & 0.65 \\
\hline & S-430 & 6.8 & 1.1 & 35.8 & 56.3 & 0.64 \\
\hline & S-530 & 6.5 & 0.8 & 41.2 & 51.5 & 0.80 \\
\hline & CF3 & 6.1 & 1.3 & 43.2 & 49.4 & 0.87 \\
\hline \multirow[t]{4}{*}{ RIO IV } & S-407 & 6.8 & 1.7 & 26.7 & 64.8 & 0.41 \\
\hline & $\mathrm{S}-430$ & 7.1 & 1.3 & 29.4 & 62.2 & 0.47 \\
\hline & S-530 & 6.8 & 1.3 & 35.9 & 56.0 & 0.64 \\
\hline & CF3 & 7.8 & 1.7 & 29.6 & 60.9 & 0.49 \\
\hline \multirow[t]{3}{*}{ PATRICIOS } & S-407 & 6.6 & 1.3 & 26.3 & 65.8 & 0.40 \\
\hline & S-530 & 5.6 & 1.1 & 30.2 & 63.1 & 0.48 \\
\hline & CF3 & 6.7 & 1.3 & 27.9 & 64.1 & 0.44 \\
\hline \multirow[t]{4}{*}{ PIGÜE } & S-407 & 6.5 & 1.7 & 21.1 & 70.7 & 0.30 \\
\hline & S-430 & 5.9 & 1.5 & 27.3 & 65.4 & 0.42 \\
\hline & S-530 & 6.3 & 2.3 & 22.4 & 69.8 & 0.32 \\
\hline & CF3 & 6.1 & 1.5 & 27.7 & 64.7 & 0.43 \\
\hline \multirow[t]{4}{*}{ NECOCHEA } & S-407 & 6.4 & 1.3 & 20.6 & 71.7 & 0.29 \\
\hline & $S-430$ & 5.8 & 1.6 & 31.0 & 61.6 & 0.5 \\
\hline & $S-530$ & 6.0 & 1.3 & 23.5 & 69.2 & 0.34 \\
\hline & CF3 & 5.0 & 2.5 & 30.0 & 62.5 & 0.48 \\
\hline
\end{tabular}


Algunas de las tendencias presentadas, fueron reportadas por otros autores (2) (3).

$\mathrm{El}$ análisis de la composición acídica se muestra en la Tabla I.

Se observa que el grado de insaturación del aceite de girasol está inversamente relacionado con la temperatura durante el período de crecimiento de la semilla. En el presente estudio, se analizó la influencia de la temperatura media ambiente en la etapa de desarrollo, teniendo en cuenta que la misma disminuye -para nuestra latitudsegún se avance de norte a sur; es decir, que la localidad I registra la mayor temperatura media ambiente $\left(28^{\circ}\right)$, siguiendo en orden decreciente las localidades II $\left(26^{\circ}\right)$, III $\left(24^{\circ}\right)$, IV $\left(23^{\circ}\right), \mathrm{V}\left(22^{\circ}\right)$ y $\mathrm{VI}\left(20^{\circ}\right)$.

El Gráfico №. 2 muestra cómo las mayores temperaturas favorecen la acumulación de ácido oleico, mientras que en las localidades más frías se obtiene un aceite de girasol rico en ácido linoleico.

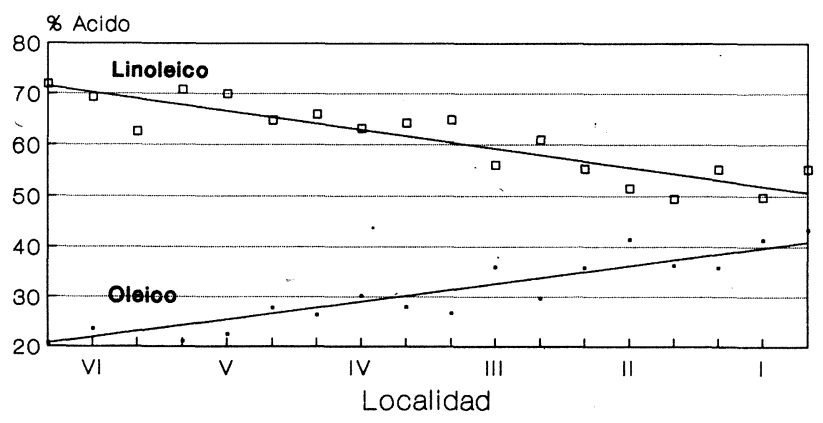

(Temperatura media ambiente ascendente) Híbridos: S-407, S-430, S-530, CF3

Gráfico № 2

Influencia de la temperatura sobre la conc. de Oleico y Linoleico

El porcentaje combinado de ácido oleico y linoleico, osciló entre $89.8 \%$ y $93.3 \%$ y no presenta influencia de la temperatura, mientras que la relación entre estos ácidos grasos varía notablemente con este parámetro, ya que se obtuvieron valores de $0.65,0.40$ y 0.28 para II, IV y VI, respectivamente, correspondientes a la Cos I y 0.69, 0.74, $0.50,0.44,0.40$ y 0.37 para las localidades I, II, III, IV, VI y $\mathrm{V}$, respectivamente, correspondientes a la Cos II (la diferencia entre los dos primeros valores está dada especialmente por las variaciones en el CF3).

Se obtuvo una fuerte y consistente correlación negativa entre las concentraciones de ácido oleico y linoleico $(r=-$ 0.996).

Estos comportamientos, ratificados para los híbridos de girasol cultivados en Argentina, coincide con lo reportado por numerosos autores (2) (4) (5) (6) (7) (8) (9) (10) (11).

La técnica utilizada para la determinación de ceras establece que la cantidad extraída corresponde al 90-95\% del total existente en la semilla (1). La Tabla II muestra los valores obtenidos (los resultados presentados son promedios de triplicados).

Resultó positiva la correlación entre el contenido de ceras en la semilla y la cantidad de cáscara $(r=+0.54)$, ten- dencia coincidente con la escasa bibliografía existente al respecto (12).

Tabla II

Contenido de ceras (\% base seca)

\begin{tabular}{lcccccc}
\hline \multirow{2}{*}{ Localidad } & \multicolumn{7}{c}{ HIBRIDO } \\
\cline { 2 - 7 } & $\begin{array}{c}\text { S-407 } \\
\%\end{array}$ & $\mathrm{~S}^{*}$ & $\begin{array}{c}\text { S-430 } \\
\%\end{array}$ & $\mathrm{~S}^{*}$ & $\begin{array}{c}\text { CF3 } \\
\%\end{array}$ & $\mathrm{~S}^{*}$ \\
\hline AVIA TERAI & 0.081 & 0.023 & 0.120 & 0.049 & 0.094 & 0.000 \\
RECONQUISTA & 0.016 & 0.023 & 0.180 & 0.021 & 0.170 & 0.021 \\
RIO IV & 0.090 & 0.000 & 0.100 & 0.007 & 0.130 & 0.014 \\
PATRICIOS & 0.100 & 0.015 & 0.120 & 0.006 & 0.130 & 0.021 \\
PIGÜE & 0.090 & 0.000 & 0.110 & 0.000 & 0.100 & 0.000 \\
NECOCHEA & 0.090 & $0.02 \dot{5}$ & 0.080 & 0.015 & 0.090 & 0.010 \\
\hline
\end{tabular}

$\mathrm{S}^{\star}$ : Desviación típica

El Gráfico № 3, muestra las variaciones en los contenidos de cera según la localidad y el híbrido; del análisis del mismo, se deduce que no es notable la influencia de la zona de cultivo sobre esta variable, ya que -excepto en Reconquista- el porcentaje de ceras no presenta variación considerable, pero sí afecta la genética de cada especie.

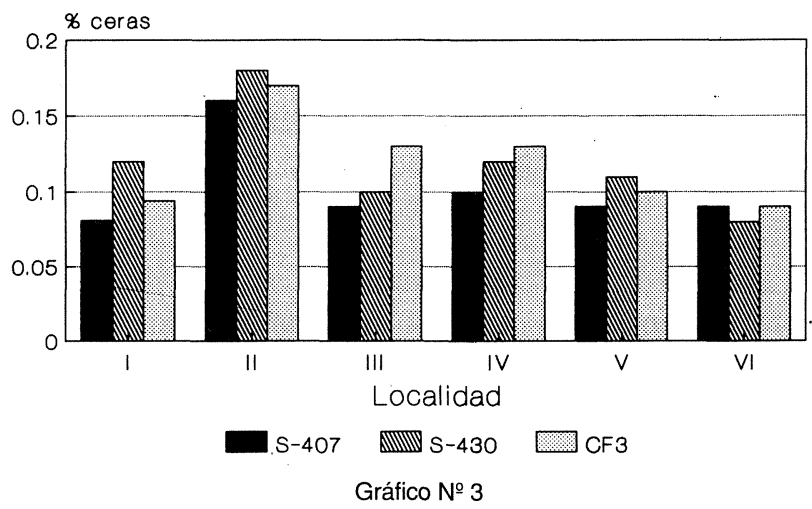

Contenido de ceras en función de la localidad

Se obtuvo tendencia de correlación positiva entre la relación \% de ceras $/ \%$ de cáscara (contenido de ceras en la cáscara) y el contenido de aceite de la semilla $(r=+0.60)$ (Gráfico nำ4).

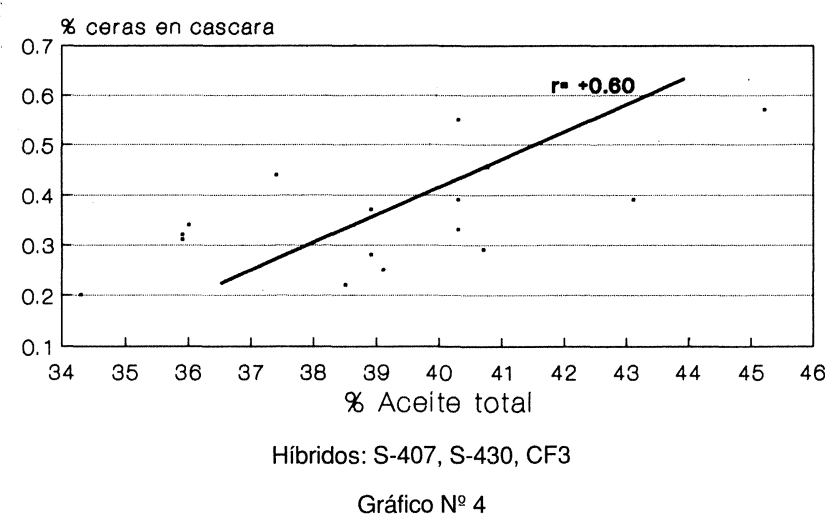

Cont. de ceras en cáscara en relación con el aceite total 
Esto último parece indicar que a mayor contenido de aceite en la semilla, como es menor la cantidad de cáscara, aumenta el porcentaje de ceras, lo que ratifica la tendencia de comportamiento puesta de manifiesto en los últimos años con el desarrollo de los nuevos híbridos de alto contenido de aceite.

\section{DISCUSION}

Ante el problema mencionado de disminución del índice de extracción en los híbridos de mayor contenido de aceite por semilla, se plantean distintas hipótesis que intentan explicar este fenómeno:

a) La separación de la cáscara al momento de preparar la muestra para la extracción, provoca pérdidas de aceite total.

b) El contenido de ceras de la cáscara, conjuntamente con la eficiencia del proceso de descascarado previo a la extracción, influyen en la pérdida de aceite crudo.

c) El incremento del contenido de aceite de los nuevos híbridos implicó cambios tecnológicos que influenciaron sobre el comportamiento del material durante la extracción.

Respecto a la primera hipótesis, las correlaciones obtenidas (cantidad de cáscara-contenido de aceite total $r=-0.70$ y $\mathrm{r}=-0.72$; cantidad de cáscara-contenido de aceite en cáscara $r=-0.31$ y $r=-0.34$, tendencias concordantes pero poco significativas en cuanto a su valor absoluto, y contenido de aceite total -contenido de aceite en cáscara $r=0.00$ ) no permiten confirmar esta hipótesis.

Las ceras presentes en el aceite crudo son eliminadas durante el proceso de refinación y durante su precipitación arrastran una cantidad considerable de aceite.

Analizando la hipótesis (b), la correlación positiva obtenida entre el contenido de cera en la cáscara y el contenido de aceite en la semilla $(r=+0.60)$, indica la importancia que tiene la separación de la cáscara en la extracción del aceite.

Por lo expuesto, al aumentar la concentración de ceras en la cáscara pequeñas cantidades de la misma -no eliminadas en el proceso de separación- pueden implicar una retención considerable de material céreo en la materia prima.

Respecto a la última hipótesis, las diferencias en los caracteres de la semilla de los híbridos modernos inducen a investigar la cinética de la extracción como así también el régimen de flujo del solvente durante la misma.

No debería descartarse una prueba a escala piloto o industrial para estudiar el mismo comportamiento, pero sobre una muestra que es en realidad una mezcla de híbridos de diferentes características genéticas y morfológicas, como ocurre en la mayoría de los procesos de extracción industrial.

\section{BIBLIOGRAFIA}

1. Morrison III,W.H.(1982),-"Rapid effective method for the reduction of waxes in sunflower seed oil".- J. Am. Oil Chemists' Soc. 59, 519-520.

2. Fernández-Martinez, J. y Domínguez-Giménez, J. (1985).-"Evaluación de la variabilidad en caracteres de la semilla de una colección mundial de girasol".- In: Proceedings 11th International Sunflower Conference, Mar del Plata, Argentina, pp 535-540.

3. Bertoni, M.H., Karman de Sutton, G., Cattaneo, P. y Gómez Artero, J.G. (1967).-"Aceites de semilla de girasol de producción nacional".- Rev. Argentina Grasas y Aceites 9 (1) 1-26.

4. Seiler, G . (1983) .-"Effect of genotype, flowering date, and environment on oil content and oil quality of wild sunflower seed" .- Crop Sci. 23,1063-1068.

5. Seiler, G. (1985).-"Interrelation of fatty acids in oil of wild annual sunflower $(H$. annuus L.)".- In: Proceedings 11 th International Sunflower Conference, Mar del Plata, Argentina, pp 529-534.

6. Skoric, D.; Vorosbaranyi, I. y Bedov, S. (1982).- "Variability in the composition of higher fatty acids in oil of sunflower inbreds with different oil contents in seed".-In: Proceedings 10th International Sunflower Conference, Australia, pp 215-220.

7. Nagao, A. y Yamazaki, N.(1983).-"Lipid of sunflower seeds produced in Japan".- J. Am. Oil Chemists' Soc. 60,1654-1658.

8. Fernández-Martinez, J., Jiménez, A., Domínguez, J., Garcia, J.M., Garces, R. y Mancha, M. (1989).-"Genetic analysis of the high oleic acid content in cultivated sunflower (H. annuus L.) -.- Euphytica 41, 39-51.

9. Harris, H.C., Mc William, J.R. y Mason, W.K. (1978).-"Influence of temperature on oil content and composition of sunflower seed .- Aust. J. Agric. Res. 29,1203-1212

10. Gupta, S.K., Wagle, D.S. y Yadava, T.P.- "Effect of environment on fatty acid composition of developing seeds of sunflower (H. annuus L.)".- 8186.

11. Robertson, J.S. (1972).-"Sunflowers: America's Neglected Crop" J. Am. Oil Chemists' Soc. 49, 239-244.

12. Morrison III, W.H., Sojka, R.E. y Unger, P.W. (1984).-"Effects of planting date and irrigation on waxes content of sunflower seed oil".-J. Am. Oil Chemists' Soc. 61,1242-1245.

(Recibido: Octubre 1992) 